\title{
Patterns of Care in Michigan Emergency Departments as Insurance Coverage Expands
}

\author{
Fatema Mamou*, Matthew Davis, Jim Collins, Jay Fiedler, Tiffany Henderson and Kiera \\ Wickliffe Berger
}

Michigan Department of Community Health, Lansing, MI, USA

\section{Objective}

The purpose of this work is to use the Michigan Syndromic Surveillance System (MSSS) to assess emergency department (ED) utilization before and after the April 2014 implementation of the Healthy Michigan Plan, an expanded Medicaid program.

\section{Introduction}

The MSSS, described elsewhere ${ }^{1}$, has been in use since 2003 and records ED chief complaint data. As of September 2014, there were 88/136 hospital EDs enrolled in MSSS, capturing $83 \%$ of the annual hospital ED visits in Michigan.

On April 1, 2014 the Healthy Michigan Plan (HMP) was launched. HMP provides healthcare benefits to low-income adult residents who do not qualify for Medicaid or Medicare. The plan incorporates both federally and state mandated Essential Health Benefits, which includes emergency services.

As insurance coverage expands, more people will have the ability to utilize the services of primary care and other providers. In particular, this will affect previously uninsured, low-income populations who are disproportionately affected by chronic disease.

We question if access to these services will affect the utilization of emergency services as more people will have a medical home to manage and prevent diseases that may otherwise become an emergent issue. Furthermore, this increased access to health care services will expand care options for urgent but not emergent issues beyond EDs. Conversely, as more people acquire health care benefits the demand for primary care services may exceed the level of access to these services which may lead to an increase of ED utilization for primary care.

\section{Methods}

Total ED visits were analyzed by month from January 2011 to date. Seasonally adjusted monthly ED visits prior to and after the HMP implementation were compared. Seasonally adjusted monthly ED visits were stratified by age and the trend of ED visits among those 18-64 years were compared to that among other age groups. Michigan Behavioral Risk Factor System data (BRFS) ${ }^{2}$ was used to categorize counties by proportion of self-reported health care coverage. Visits were assigned one of four health care coverage categories from low to high based on county of residence. Trends in visits for each category were analyzed.

Select chief complaints that represent illnesses or medical needs that could be addressed by a primary care provider were categorized as primary care sensitive. Seasonally adjusted monthly totals for these complaints were analyzed for trends before and after the HMP launch. These visits were further stratified by time of visits to observe the changes in the volume of these visits during times when primary care access is reduced or unavailable.

\section{Results}

A total of 76 facilities have been used in the analysis with an average of 330,000 ED visits per month. As of August 2014 over 355,000 Michigan residents have enrolled in HMP. Analyses are ongoing, but preliminary data as of August 2014 show no significant difference observed in seasonally adjusted total ED visits per month after the launch of the HMP compared to before. Similarly, no difference has been observed by age group. A small increasing trend has been observed among visits from people residing in counties that had a low proportion of health insurance coverage based on BRFS data.

No significant difference in the trend of primary care sensitive chief complaints has been observed.

\section{Conclusions}

Long term data analyses are needed to evaluate changes in ED utilization as a result of expanded health care coverage. Ongoing analyses of this data will be conducted as more and more eligible Michigan adults enroll into the HMP.

Lack of a medical home combined with decreased financial barriers may lead new HMP enrollees to seek medical care at EDs.

To validate the potential increasing trends observed in counties with previously reported low health insurance coverage ${ }^{2}$, ED visits will be compared to HMP enrollment data. Monthly trends of ED visits among counties with high enrollment into the plan will be compared to ED visits among counties with lower enrollment to assess for differences in ED utilization before and after the launch of the HMP.

\section{Keywords}

Sydromic surveillance; Health insurance coverage; ED utilization

\section{References}

1. Sheline KD. Evaluation of the Michigan Emergency Department Syndromic Surveillance System. Advances in Disease Surveillance. 2007; 4: 265

2. Michigan Department of Community Health (MDCH). Health Indicators and Risk Estimates by Community Health Assessment Regions \& Local Health Departments, State of Michigan, Selected Tables 2011 - 2013. Lansing (MI): Michigan Behavioral Risk Factor Survey; July 7, 2014. Table 7, No Health Care Coverage Among Those Aged 18-64 Years; p. 18-19.

*Fatema Mamou

E-mail:mamouf@michigan.gov 